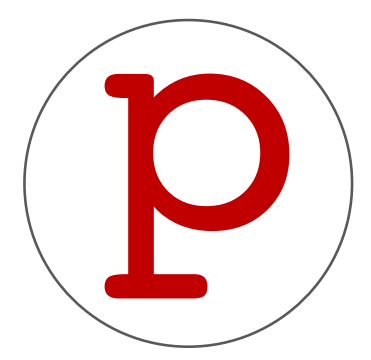

polis isemiee

$$
\mid
$$

I

2020 


\section{pol isemie}

I

\section{0}

Direzione

Stefano Milonia (University of Warwick)

Comitato scientifico

Niccolò Scaffai (Università degli Studi di Siena)

Carlo Pulsoni (Università di Perugia)

Luigi Marinelli (Università di Roma Sapienza)

Giulia Bassi (Università degli Studi di Siena)

Mario Cianfoni (Università di Roma Sapienza)

Costantino Turchi (Università di Roma Sapienza)

\section{Redazione}

Mattia Caponi (Università di Roma Sapienza)

Stefano Bottero (Università di Roma Sapienza)

Samuele Maria Visalli (Università di Roma Sapienza)

Giuseppe Giorgio Tranchida (Alma Mater Studiorum Università di Bologna)

Arianna Saggio (Università di Roma Sapienza)

Giulia Boitani (University of Cambridge)

Giulia Greco

Alessandra Frustaci

Polisemie è una rivista annuale pubblicata dalla University of Warwick Press.

Gli articoli pubblicati nella sezione Saggi sono sottoposti a double-blind peer review.

Licenza Creative Commons - Attribuzione (CC-BY 4.0).

ISSN: 2634-1867

DOI: 10.31273/polisemie.v1

Copertina: Alessia Provinciali 


\section{EDITORIALE}

La rivista Polisemie nasce dall'iniziativa di giovani ricercatori e studenti dell'Università di Roma Sapienza, dell'Università degli Studi di Siena, dell'Alma Mater Studiorum di Bologna e dell'Università di Warwick, per approfondire lo studio della poesia contemporanea in Italia e fuori. Ciò che spinge alla fondazione di questa rivista è la volontà di capire il presente, nelle sue forme che a priori non possono che apparire irriducibilmente caotiche, con l'obiettivo a lungo termine di tracciare i contorni del fenomeno della scrittura poetica in un sistema ordinato anche se provvisorio e parziale.

Il programma della rivista è di leggere con la stessa attenzione gli autori di quello che si delinea come un canone degli anni Duemila e quelli il cui nome è ancora sconosciuto, allo scopo di farne materia di studio accademico. Di fatto, descrivere e comprendere fenomeni complessi è compito della scienza, sia pure essa scienza letteraria, senza eccezione per il fenomeno della poesia del presente. Sebbene la scienza letteraria sia incardinata, nella sua più ampia applicazione, fra le scienze storiche, essa non è per questo ontologicamente tale. La ricerca legata alla rivista Polisemie si propone come ricerca sul campo, in quanto unico metodo di analizzare un organismo che non abbia concluso il suo ciclo vitale e il cui inalienabile valore come oggetto di studio è legato proprio a tale vitalità. In un'epoca in cui il prestigio legato alla scrittura poetica segue una direttrice più negativa che in nessun altro tempo, offrire una riflessione di carattere scientifico è un contributo significativo per riaffermare da una parte la dignità letteraria della scrittura in versi e dall'altra la capacità della ricerca letteraria di avere un contatto e un impatto sulla società e sulla realtà attuale.

Chi si interessa di letterature prodotte in epoche più lontane può sopperire al desiderio di interrogare l'autore di cui si sta occupando in quel momento (anonimo, semisconosciuto, o deceduto da vari secoli) con vaste bibliografie e non tralascia nessuna fonte che possa offrire anche il minimo indizio sul poeta o sull'opera. La ricerca che si occupa del presente pone condizioni opposte: non ci sono bibliografie a incamminarci, se non anch'esse esplorative. Il poeta, al contrario, è vivo e può rispondere. Il dialogo si pone allora come prima fonte utile a tracciare una mappa per la ricerca. A questo scopo, per quanto possibile, gli articoli della rivista sono accompagnati da interviste, che vanno considerate come un valore aggiunto al contributo scientifico. Sebbene l'oggetto di studio dovrebbe restare sterile e 
distaccato dall'osservatore, da cui è necessariamente influenzato, una critica che sceglie di tralasciare alcune fonti ancor prima di consultarle rischia di omettere elementi fondamentali e perdere l'occasione di giungere a importanti risultati. Far finta che il testo non abbia un autore vuol dire rinunciare alla propria capacità di discriminare tra autore e testo. In quanto "prima persona informata sui fatti", l'autore è un testimone che non può essere ignorato e, direttamente $\mathrm{e}$ indirettamente, è un tassello importante nella formazione dell'interpretazione critica. Questo aspetto, che potrà risultare banale, ma di cui molti riconosceranno l'innovazione metodologica, è centrale in un'inchiesta su materiale inedito, che rischia di essere mal interpretato se sottoposto a categorie ad esso non applicabili. Infine, per la sua maggiore fruibilità, la registrazione di un'intervista può aspirare a coinvolgere un maggior numero di lettori e contribuire così a un circuito virtuoso di comprensione e lettura.

La rivista che si apre con questo primo fascicolo è il cardine delle iniziative legate a Polisemie. Attorno ad essa sono stati realizzati eventi dedicati alla poesia del presente, nel tentativo di creare un ponte tra la scrittura accademica e un pubblico più ampio di interessati. Dal 2019, Polisemie organizza cicli di incontri tra poeti e critici all'Università di Roma Sapienza, dove, il 24 maggio 2019, ha avuto luogo il primo Festival di poesia iper-contemporanea. Nella stessa sede avrà luogo il Convegno internazionale VentiVenti: Per un'interpretazione della poesia d'inizio secolo. 